\title{
Neuron Model of Interprofessional Education and Evidence-Based Practice
}

\author{
Dixon Thomas, $\mathrm{PhD}^{1}$; Jason C. Cooper, PharmD²; Mark Maas, PharmD²
}

${ }^{1}$ Gulf Medical University, Ajman, United Arab Emirates; ${ }^{2}$ Medical University of South Carolina, Charleston, SC, United States

\begin{abstract}
Interprofessional education (IPE) and evidence-based practice (EBP) are relatively new concepts in health professions education in many parts of the world. These critical reforms are implemented with great effort. As clinical practice has become more collaborative and evidence-based, teamwork and research need to be well integrated in the curriculum. However, many stakeholders struggle to visualize the work of IPE and EBP in the context of health professions education and practice. The Neuron Model, using parts of the neuron, is designed to detail how IPE and EBP integrate in health professions curriculum design or reveal a hidden curriculum. Evidence-based interprofessional care has been implemented with limitations in academic health systems. Lack of a common understanding of how it works is a limitation. The neuron model thus aims to visualize IPE and EBP in health professions education and practice.
\end{abstract}

Keywords: health professions education, interprofessional education, Neuron Model, evidence-based practice

\section{INTRODUCTION}

Interprofessional education (IPE) and evidence-based practice (EBP) are implemented with significant effort in health professions' education. Professionals from different specializations may need further clarity to conceptualize these curriculum trends correctly. When properly implemented, the educational benefits of IPE and EBP can be realized. ${ }^{1,2}$ This commentary aims to explain how to visualize IPE and EBP in health professions education using the Neuron Model.

Before the separation of health professions, practitioners held a set of broader, dynamic competencies. Specializations within the professions have both enabled and handicapped practitioners at the same time. Specializations advance knowledge, but limits understanding to a narrow field of practice. Specializations even separated basic and clinical sciences within a profession. The basic sciences could be applied in the context of practice. These courses support the basis for a comprehensive understanding of therapy, thus integrating sciences to practice is an ongoing reform in health professions curricula. ${ }^{3}$

Health professions education is continually transforming to impart improved clinical knowledge, skills, and attitudes for graduates to be ready to practice upon completion of a program. However, just having knowledge is not enough. There is a greater need for practice-based skills, including interprofessional care and research. For example, students should be exposed to and taught about collaborative practice

Corresponding author: Dr. Dixon Thomas, PhD

Associate Professor \& Chair, Department of Pharmacy Practice

College of Pharmacy, Gulf Medical University, UAE

Director, Center for Drug Information \& Evidence-Based

Pharmacy, Thumbay University Hospital, UAE

Mobile: +971557540701

Email: dr.dixon@gmu.ac.ae, dixon.thomas@gmail.com environments where the therapy of complex medical conditions require teamwork skills and healthy attitudes towards patients and other healthcare providers. ${ }^{1}$

Such IPE activities should start earlier in the curriculum and may lead to improved student engagement. ${ }^{4}$ Letting students find the relevance of their studies, while imagining themselves as future health professionals, should happen from the beginning. ${ }^{5}$

Health professionals are used to studying and practicing in their professional domains. It can be difficult for many professionals to visualize the work of IPE and EBP in health professions education and practice. To move forward, a model that provides room for independent research in a professional's area of practice, while being collaborative, is essential.

\section{THE NEURON MODEL IN IPE AND EBP}

The integrate-and-fire model is commonly used in analyzing the behavior of neural systems. This model explains many of the essential features of neural processing. ${ }^{6}$ This commentary is inspired by this integrate-and-fire model. It uses the structure of a neuron for visualizing the work of IPE and EBP in health professions education and practice.

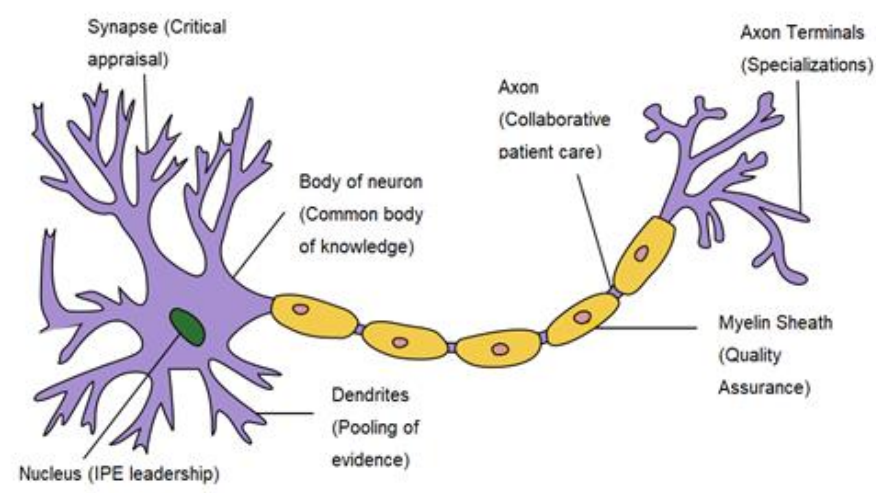

Figure 1. Neuron model in Interprofessional Education and Evidence-Based Practice (Adapted from Jarosz Q. Wikipedia) 
Do only mathematicians perform calculations? The answer is no. Similarly, not only pharmacists provide advice on medications. Health professionals excel in their own way, but they may occasionally fulfill the roles of one another. At some part of their practice, when a group of health professionals work together for the benefit of the patient, they resemble an axon. At other times, when they travel on different routes to specialize and provide for unique needs, they are like axon terminals and dendrites. Crossing the synapse is required to pool these unique skills into a collective body of knowledge that is the body of neuron. For example, the development of any clinical guidelines or a new drug discovery involves contributions from multiple health professionals. Once the guidelines are implemented or the new drug is approved, all professionals apply it with a common understanding.

Represented by the axon in the Neuron Model, health professions unite together through education and practice to optimize patient outcomes. Delivery of patient care is fast and efficient when a quality assurance system (myelin sheath) protects the process. Preparation and readiness for appropriate patient care across the whole health system takes significant effort through education of healthcare professionals. Once the system is ready, via training and implementation, it works quickly and efficiently. For these reasons, many quality assurance practices and accreditation standards in health professions education recommend IPE. ${ }^{7}$

Patient care, collaborative or not, should be evidence-based. Evidence generation and utilization occur at different levels of practice based on resources and requirements. In terms of the Neuron Model, axon terminals represent different professionals, who specialize in their area of practice. Conducting research or even reading research is performed by a single professional, a group of similar professionals, or professionals from different professions with or without students. Interpretation of these pieces of evidence occurs through the peer-review process in formal or informal journal clubs or case discussions. This process of crossing synapses in the Neuron Model represents critical appraisal skills in screening the best quality evidence. Healthcare professionals are expected to have proficiency in research interpretation, so its inclusion in curricula is essential.

Students who are introduced to the research process in undergraduate courses enhance their understanding and interest in practice-based research and can improve their confidence in research areas. ${ }^{8}$ However, significant assistance is required initially for students to perform original research. If students do not receive adequate training, they may feel lost and develop a generalized disdain towards research. ${ }^{9}$ Studying different research methods will help students improve their interpretation and application of clinical trial design and outcomes. ${ }^{10}$ If students immerse themselves in multiprofessional research during the health professions curriculum, they will be able to collaborate more effectively with other providers upon their entry into practice.

Pooling best quality pieces of evidence through the dendrites of different professions in the Neuron Model is not enough for collaborative practice. Productive deliberations should happen in the body of neuron among different healthcare professionals. The integration of pieces of evidence from different professions into common clinical guidelines requires extensive effort, however.

Professionals are comfortable with the way they are used to working, especially highlighting the relevance of their profession on patient care: prescribing (doctors), injections (nurses), dispensing (pharmacists), tests (laboratory technicians), or economics and management (analysts/managers). Thus, many tend to practice and study in isolation. Too much isolation, however, can be problematic. In order to avoid being completely antiquated, professionals tend to overlap on duties of other professions. That is why IPE is so critical; it crosses traditional boundaries and redefines professional identities to improve patient care. While preserving the image of a singular profession, a common image must also be embraced. Having an evidence-based approach to this learning model is crucial, as it generates ideas that lead to further research and ultimately new evidence for practice integration.

The nucleus in the Neuron Model shows the role of leadership in coordinating IPE and EBP. Required leadership should be available from different professions and should facilitate collaborative and evidence-based learning within the practice environment. It should also function to be sensitive to the needs of patients, as well as the dynamics of stakeholders involved, in order to determine and implement what is good for all.

Hopefully, the illustration of IPE and EBP using the Neuron Model helps to visualize how IPE and EBP work together in harmony. It may also address the concerns of many who are hesitant to initiate IPE or reconsider their failed attempts and continue doing IPE with confidence.

\section{CONCLUSION}

Ultimately, patients are the beneficiaries of health professions education. Though many believe IPE and EBP are reasonable, some struggle to visualize the interface of the two. The Neuron Model illustrates IPE in the context of collaborative patient care, while preserving EBP. As it shows, collaborative practice cannot deliver patient care without allowing specializations inherent within different health professions. We recommend future research on the impact on IPE awareness among different stakeholders, after learning about the Neuron Model.

Conflicts of Interest: None

Funding/Support: None 


\section{REFERENCES}

1. Pearson ML, Hubball HT. Curricular integration in pharmacy education. Am J Pharm Educ. 2012;76(10):Article 204. doi: 10.5688/ajpe7610204

2. West C, Graham L, Palmer RT, et al. Implementation of interprofessional education (IPE) in 16 US medical schools: Common practices, barriers and facilitators. J Interprof Educ Pract. 2016;4:41-9. doi: 10.1016/j.xjep.2016.05.002

3. Husband AK, Todd A, Fulton J. Integrating science and practice in pharmacy curricula. Am J Pharm Educ. 2014;78(3):Article 63. doi: 10.5688/ajpe78363

4. O'Neil-Pirozzi TM, Musler JL, Carney M, Day L, Hamel PC, Kirwin J. Impact of early implementation of experiential education on the development of interprofessional education knowledge and skill competencies. J Allied Health. 2019;48(2):E53-E59.

5. Thomas D, Marriott J, Vadlamudi R, Efendie B, Maine LL. Introduction to Clinical Practice, Research, and Pharmacy Education. In: Clinical Pharmacy Education, Practice and Research. 2019:1-9. doi: 10.1016/B9780-12-814276-9.00001-5

6. Burkitt AN. A review of the integrate-and-fire neuron model: I. Homogeneous synaptic input. Biol Cybern. 2006;95(1):1-9. doi: 10.1007/s00422-006-0068-6
7. Health Professions Accreditors Collaborative. Guidance on developing quality interprofessional education for the health professions. 2019. Chicago, IL: Health Professions Accreditors Collaborative. Available at: https://healthprofessionsaccreditors.org/wpcontent/uploads/2019/02/HPACGuidance02-0119.pdf. Accessed August 13, 2020.

8. Perez $A$, Rabionet $S$, Bleidt $B$. Teaching research skills to student pharmacists in one semester: an applied research elective. Am J Pharm Educ. 2017 Feb 25;81(1): Article 16. doi: 10.5688/ajpe81116

9. Brennan E, Cooper JC, Davis A. Evidence-Based Practice: Use in Answering Queries and Developing Systematic Reviews. In: Clinical Pharmacy Education, Practice and Research 2019:163-176. doi: 10.1016/B978-0-12-814276-9.00011-8

10. Maharajan MK, Rajiah K, Tam AM, Chaw SL, Ang MJ, Yong MW. Pharmacy students' anxiety towards research during their undergraduate degree; How to reduce it? PLoS ONE. 2017;12(4): e0176095. doi: 10.1371/journal.pone.0176095 